\title{
On the cosmic ray cross-field diffusion for solar wind turbulence
}

\author{
G. Michałek ${ }^{\star}$ \\ 1 Center for Solar Physics and Space Weather, Department of Physics, The Catholic University of America, \\ Washington, DC 20064, USA \\ 2 Obserwatorium Astronomiczne, Uniwersytet Jagielloński, ul. Orla 171, 30-244 Kraków, Poland
}

Received 8 February 2001 / Accepted 12 June 2001

\begin{abstract}
The cross-field diffusion coefficient of charged particles in the presence of pure isotropic Alfvén waves, pure isotropic magnetosonic waves and their mixture is considered using Monte Carlo particle simulations. We show that the cross-field diffusion coefficient $\kappa_{\perp}$ strongly depends on the assumed spectrum and amplitude of MHD turbulence but much less on the type of waves considered. It is demonstrated that transport perpendicular to the average magnetic field is mostly determined by compound diffusion which combines the effects of scattering off magnetic irregularities with magnetic braiding. The ratio $\kappa_{\perp} / \kappa_{\|} D_{\mathrm{M}} \approx$ const. is independent of rigidity over the wide range considered.
\end{abstract}

Key words. cosmic rays - magnetohydrodynamics (MHD) - turbulence - solar wind

\section{Introduction}

Observations of solar energetic particle events confirm that energetic particle transport in the heliosphere must be considered as a diffusive process. Understanding the mechanism by which particles are scattered in cosmic plasma continues to be one of the most important problems of modern cosmic-ray physics. The main physical process underlying the diffusive propagation is assumed to be pitch-angle scattering by magnetic fluctuations imposed on the large-scale magnetic field. It is usually analyticly described by the quasi-linear theory (QLT; cf. Jokipii 1966; Hasselman \& Wibberenz 1968; Schlickeiser 1989). The quasi-linear theory for the parallel diffusion $\left(\kappa_{\|}\right)$of cosmic rays (CR) appears to be reasonably well developed, in contrast to the perpendicular diffusion coefficient $\left(\kappa_{\perp}\right)$. That coefficient is one of the most crucial parameters influencing the solar modulation of CR. In spite of its importance, there is no fundamental theory of perpendicular transport. It was only well established for hard-sphere scattering in a magnetized plasma (Gleeson 1969). Other analytical considerations based on Foker-Planck theory (Forman et al. 1974; Jokipii \& Parker 1969) were limited to a weak perturbed slab magnetic turbulence. However, recent results indicate that in the case of solar wind we encounter complex high-amplitude turbulence (Bieber et al. 1996). What is more, Giacalone \& Jokipii (1994) proved that diffusion across the mean magnetic field needs a three dimensional structure for the

\footnotetext{
* e-mail: michalek@oa.uj.edu.pl
}

turbulent magnetic field. Similarly, Jokipii \& Kota (1999) showed that the method based on the velocity correlation function, developed by Kubo in 1957 to evaluate spatial diffusion coefficients, cannot be used for compound diffusion, in which the particles are scattered back and forth along spreading magnetic field lines. In two papers (Michałek \& Ostrowski 1997, 1998) it was shown that particle transport across magnetic field is a very complex process. It depends, in very complicated way, on resonant interaction with MHD turbulence, diffusion of magnetic field lines and drifts due to gradients and curvature of magnetic irregularities. Recently, Giacalone \& Jokipii (1999, $\equiv$ GJ99) presented numerical simulations of perpendicular and parallel diffusion coefficients in the presence of a stationary irregular magnetic field. They showed that the cross-field diffusion could be larger than the prediction of classical scattering but smaller than the prediction of the quasi-linear theory. The transport of charged particles across the average magnetic field must be considered as the superposition of two fundamental effects. The first is scattering at an irregular magnetic field, the second is caused by wandering or braiding of the magnetic field lines. Depending on the efficiency of these processes the transport of charged particles normal to the mean magnetic field may be described by ballistic motion, subdiffusive motion or compound diffusion (Duffy et al. 1995). A Markovian process involving diffusion along a magnetic field, that is itself wandering, is regarded as compound diffusion. In many astrophysical circumstances transport across the mean magnetic field could be controlled by this process. 
The aim of the present paper is to study $\kappa_{\perp}$ in the heliosphere at $1 \mathrm{AU}$. We consider the particle transport in the presence of different amplitude MHD turbulence models involving pure isotropic Alfvén waves, pure isotropic magnetosonic waves or their mixture. The influence of the wave amplitude and the wave spectrum on charged particle transport is also considered. The Monte Carlo simulations performed involve derivations of particle trajectories are described in Sect. 2. Next, in Sect. 3 we present results of simulations and in Sect. 4 summarize the results.

\section{Numerical modeling}

Let us consider an infinite region of tenuous plasma with a uniform mean magnetic field along the $z$-axis $\left(\boldsymbol{B}_{\mathrm{o}}=\right.$ $\left.B_{\mathrm{o}} \hat{z}\right)$, perturbed with propagating MHD waves. In this respect our model substantially diverges from the solar wind spiral-like configuration of the mean magnetic field. Test particles (protons) are injected into turbulent magnetized plasma and their trajectories are followed by integrating particle equations of motion in space and momentum. By averaging over a large number of trajectories one derives the cross field diffusion coefficient field $\kappa_{\perp}$. In simulations we usually used 200 particles with the same initial velocity $v_{\text {ini }}$ in an individual run. The 4 th-order RungeKutta integration code with a constant integration step was used. The number of particles engaged into simulations is limited due to extensive integration time for individual trajectories. Simulations with much bigger number of particles for a few particular cases did not reveal any significant difference. A number of tests of the code were performed. They included standard checks of the applied random number generator. The accuracy of a long time integration was checked by repeating computations with shorter time steps. Also, for static perturbations, the particle energy was conserved within the required accuracy.

\subsection{Wave field models}

In the simulations the turbulence is represented as a superposition of either isotropic Alfvén waves, or of isotropic fast mode magnetosonic waves, or as a mixture of isotropic fast mode and Alfvén waves. For any individual particle a separate set of wave field parameters is selected. As a result all averages taken over particles include also averaging over multiple magnetic field realizations. We consider wide wave spectra and thus the next important question is about the number of waves used in simulations. The particle motion can become diffusive only if the trapping width, which is proportional to the square root of the wave amplitude (Karimabadi \& Menyuk 1991), of adjacent waves overlap. The waves used in the simulations have to constitute such a continuous spectrum of resonances. In order to select a sufficient number of waves providing the required resonance overlap, we performed simulations with a growing number of waves in our wide-range spectrum. For a few hundred waves applied, the generated space particle distributions were very well fitted with Gaussian functions, not revealing any discrete resonances in the wave spectrum. Thus we decided to build the considered wave field of still larger number of 768 wave modes. In the case of a mixture of MHD waves the same numbers of isotropically distributed magnetosonic waves and Alfvén waves are used. For an individual $i$ th wave, the magnetic field fluctuation vector $\delta \boldsymbol{B}^{(i)}$ is given in the form:

$\delta \boldsymbol{B}^{(i)}=\delta \boldsymbol{B}_{\mathrm{o}}^{(i)} \sin \left(\boldsymbol{k}^{(i)} \cdot \boldsymbol{r}-\omega^{(i)} t+\Phi^{(i)}\right)$.

The wave parameters - the wave vector $\boldsymbol{k}$ and the wave amplitude $\delta B_{\mathrm{o}}$ are drawn in a random manner from the Kolmogorov wave spectrum $F(k) \propto k^{-5 / 3}$, and the phases $\Phi$ are random. Additionally, the selected wave amplitudes are scaled to keep constant the model parameter $\delta B$ :

$$
\left[\sum_{i=1}^{768}\left(\delta B_{\mathrm{o}}^{(i)}\right)^{2}\right]^{1 / 2} \equiv \delta B
$$

The dispersion relations in the low- $\beta$ plasma for the Alfvén, $\omega_{\mathrm{A}}^{2}=\boldsymbol{k}_{\|}^{2} V_{\mathrm{A}}^{2}\left(\boldsymbol{k}_{\|}\right.$- a wave vector parallel to the mean magnetic field), and the magnetosonic waves, $\omega_{\mathrm{M}}^{2}=k^{2} V_{\mathrm{A}}^{2}$, provide a respective frequency $\omega$ for a given wave vector. For the fast mode and Alfvén waves the direction of $\boldsymbol{V}$ was selected randomly from an isotropic distribution. In the simulations we adopted the Alfvén velocity characteristic for the solar wind conditions, $V_{\mathrm{A}}=5 \times 10^{-4} c$ (Goldstein et al. 1995). Additionally, we assume that the mean magnetic field has a magnitude of $5 \mathrm{nT}$ at $1 \mathrm{AU} . \delta B$ is given in units of $B_{0}$.

We consider three types of wave spectra. In the first "wide range spectrum" (hereafter referred to as the "wide spectrum" $\equiv \mathrm{W}$ ) the wave vectors are drawn in a random manner (on a logarithmic scale) from the broad wave vector range $\boldsymbol{k} \in\left(\boldsymbol{k}_{\mathrm{min}}=0.1 \boldsymbol{k}_{\mathrm{res}}^{\mathrm{min}}, 10.0 \boldsymbol{k}_{\mathrm{res}}^{\max }\right)$, where $\boldsymbol{k}_{\mathrm{res}}^{\min }$ and $\boldsymbol{k}_{\mathrm{res}}^{\max }$ are respectively the resonant wave vectors in the mean magnetic field $\left(<B>\equiv<\sqrt{B_{\mathrm{o}}^{2}+\delta B^{2}}\right)$ for the fastest and slowest particles considered in simulations. This spectrum seems to be the most reasonable for turbulence in a solar wind. The second one is a "cutoff spectrum" (hereafter referred to as a "cutoff spectrum" $\equiv \mathrm{C})$ with the wave vectors drown from the range $\boldsymbol{k} \in\left(\boldsymbol{k}_{\min }=0.1 \boldsymbol{k}_{\mathrm{res}}^{\min }, \boldsymbol{k}_{\mathrm{c}}^{\max }\right)$, where $\boldsymbol{k}_{\mathrm{c}}^{\max }$ is the resonance wave vector for particles with a rigidity of $1 \mathrm{GV}$. Such a cutoff in the turbulence spectrum, near the gyroresonance for protons, was postulated by Ragot (1999). By choosing such a spectrum we can study the influence of nonresonant scattering on particle transport. Finally we introduce a "narrow range spectrum" (hereafter this spectrum is referred to as a "narrow spectrum" $\equiv \mathrm{N}$ ) with turbulence power concentrated in the resonance range for each particle energy. In this case the wave vectors are drown in a random manner from a narrow band near the resonant wave vector for a given initial particle velocity $v_{\text {ini }}$, $\boldsymbol{k} \in\left(\boldsymbol{k}_{\min }=0.1 \boldsymbol{k}_{\mathrm{rin}}^{v_{\mathrm{ini}}}, \boldsymbol{k}_{\max }=10.0 \boldsymbol{k}_{\mathrm{res}}^{v_{\mathrm{ini}}}\right)$, where $\boldsymbol{k}_{\mathrm{res}}^{v_{\text {ini }}}$ is the resonant wave vector for particles with the given velocity $v_{\text {ini }}$. It must be stressed that spectrum of this last type is used for comparison only. For illustration, in the Fig. 1 


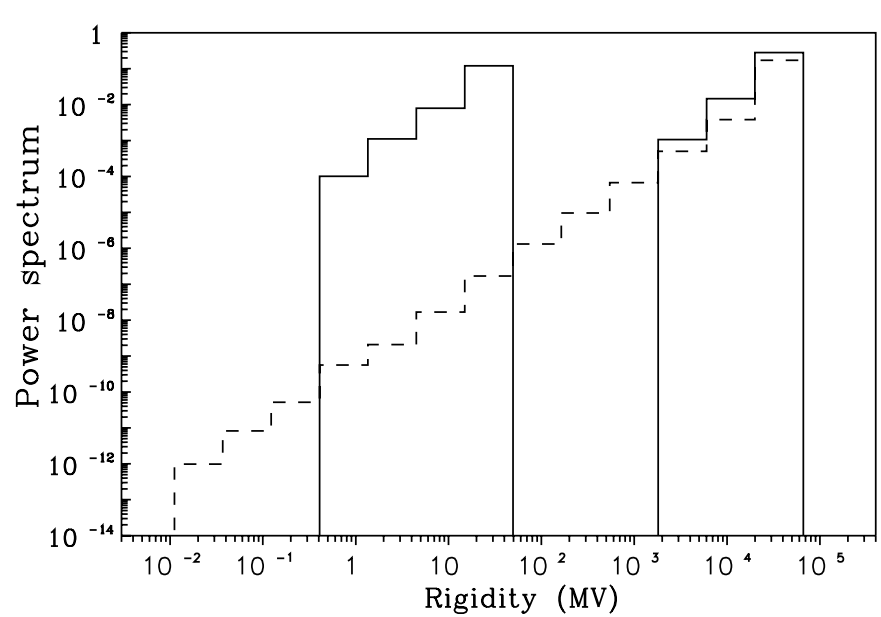

Fig. 1. Simulated wave power spectra versus respective resonant particle rigidity. The results for a wide spectrum, a cutoff spectrum and an example of a narrow spectrum for particles with rigidity about $4 \mathrm{MV}$ are presented by dashed, thick solid and thin solid lines respectively.

simulated power spectra of waves versus respective particle rigidity are presented. In our considerations, velocities of protons range from the nonrelativistic $v_{\text {ini }}=10^{-4} c$ (equivalent to rigidity $R=0.09 \mathrm{MV}$ ) up to the relativistic ones, $v_{\text {ini }}=0.999 c\left(R=2 \times 10^{4} \mathrm{MV}\right)$. In the discussion below we will examine energetic protons propagating in nine different turbulent field models: W, C and N spectra for isotropic Alfvén waves, isotropic fast mode waves and their mixture in equal proportions.

\section{Results of simulations and discussion}

In Fig. 2, simulated values of the cross field diffusion coefficient versus rigidity for all considered models are shown. From top to bottom of the picture, in the respective panels, the results for the pure Alfvén waves, for mixture of waves and for magnetosonic waves are presented. From left to right the simulated values of $\kappa_{\perp}$ for the N, C and $\mathrm{W}$ spectra are given. One should remember that the results for $\mathrm{N}$ spectra are obtained with a different spectrum for each point, and they are provided for comparison only. Additionally, the diffusion coefficients of the magnetic field $D_{\mathrm{M}}=\left\langle L^{2}>/ 2 s\right.$ (where $L$ is separation of adjacent field lines and $s$ is the distance measured along the field line) for all considered models (for $\delta B=0.3$ ) are given by thin solid lines. In the figure one may observe that simulated values of $\kappa_{\perp}$ for $\mathrm{C}$ and $\mathrm{W}$ spectra are constant in the low and middle rigidity range and increase in the high rigidity range. For models with the $\mathrm{N}$ spectrum a clear increase of the cross field diffusion coefficient is observed over the whole considered rigidity range. These general trends do not depend on the type of MHD waves used for simulations. The second obvious feature seen in the picture are much bigger cross field diffusion coefficients for the models with larger wave amplitude of $\delta B=0.6$ in comparison to the ones with $\delta B=0.3$. For models with the $\mathrm{N}$ spectrum a slight increase of $D_{\mathrm{M}}$ (about one order in magnitude) with rigidity is observed. This is caused by a steeply growing number of longer waves in the wave spectrum range taken for simulations when the rigidity of the particles considered increases. The same reason, a larger number of longer waves, is responsible for a small increase of $D_{\mathrm{M}}$ for models with the $\mathrm{C}$ spectrum in comparison to the models with $\mathrm{W}$ spectrum. In Fig. 3 the simulated values of the parallel diffusion coefficient versus rigidity for all considered models are presented. From top to bottom of the figure, in the respective panels, the results for pure Alfvén waves, for a mixture of waves and for pure magnetosonic waves are presented. From left to right the simulated values of $\kappa_{\|}$for the N, C and W spectra are given. Additionally, the ratio $\kappa_{\perp} / \kappa_{\|} D_{\mathrm{M}}$ for all considered models (for $\delta B=0.3$ ) are given by thin solid lines. The same trends as in the case of $\kappa_{\perp}$ are observed for $\kappa_{\|}$but now the parallel diffusion coefficients are much bigger for the models with a smaller wave amplitude of $\delta B=0.3$ in comparison to the ones with $\delta B=0.6$. For all spectra models the ratio $\kappa_{\perp} / \kappa_{\|} D_{\mathrm{M}}$ is roughly independent of energy. A detailed discussion of $\kappa_{\|}$presented in Fig. 3 was performed by Michalek \& Ostrowski (2001). However, it is important to note that Schlickeiser \& Miller (1998) have shown that the dominant wave-particle interaction with oblique fast magnetosonic wave is transit time damping via the $n=0$ resonance. The parallel diffusion coefficient and the related mean free paths, in the presence of massive scattaring due to transit-time damping, are determined by the gyroresonance interaction from the small pitchangle interval. In specific plasma turbulence consisting of a mixture of isotropic fast-mode waves and slab Alfvén waves, the mean free path could be independent of rigidity. Simulations for a highly perturbed magnetic field presented in Fig. 3 show that particle propagation along the mean magnetic field is mostly determined by the gyroresonance scattering and, at small rigidity, by low efficiency nonresonant interaction, but not transit-time damping.

The most striking feature emerging from the both pictures are that the cross field diffusion coefficient is smaller by about two orders of magnitude with respect to the parallel diffusion coefficients but strictly follows their behaviour over the wide rigidity range considered. It seems that parallel transport could be the most important parameter determining $\kappa_{\perp}$. Such a tendency could appear when the transport across magnetic fields is controlled by compound diffusion. In such a Markovian process, particles diffuse along a magnetic field which is itself wandering. In contrast to sub-diffusive motion a particle can escape and restart motion in a new coherent patch of field lines. In this collisional transport regime $\kappa_{\perp} \approx D_{\mathrm{M}} \kappa_{\|} / \lambda_{\mathrm{L}}$, where $\lambda_{\mathrm{L}}$ is the Lyapunov length describing the exponential separation of adjacent field lines (Duffy et al. 1995). So then, if the cross field diffusion is described by compound diffusion, the ratio $\kappa_{\perp} / \kappa_{\|} D_{\mathrm{M}}$ should be $\approx$ const. The values of $\kappa_{\perp} / \kappa_{\|} D_{\mathrm{M}}$ received for the models $\mathrm{W}, \mathrm{C}$ and $\mathrm{N}$ are almost independent of rigidity.

For models with the $\mathrm{C}$ spectrum, where in the low rigidity range particles could propagate without resonant 


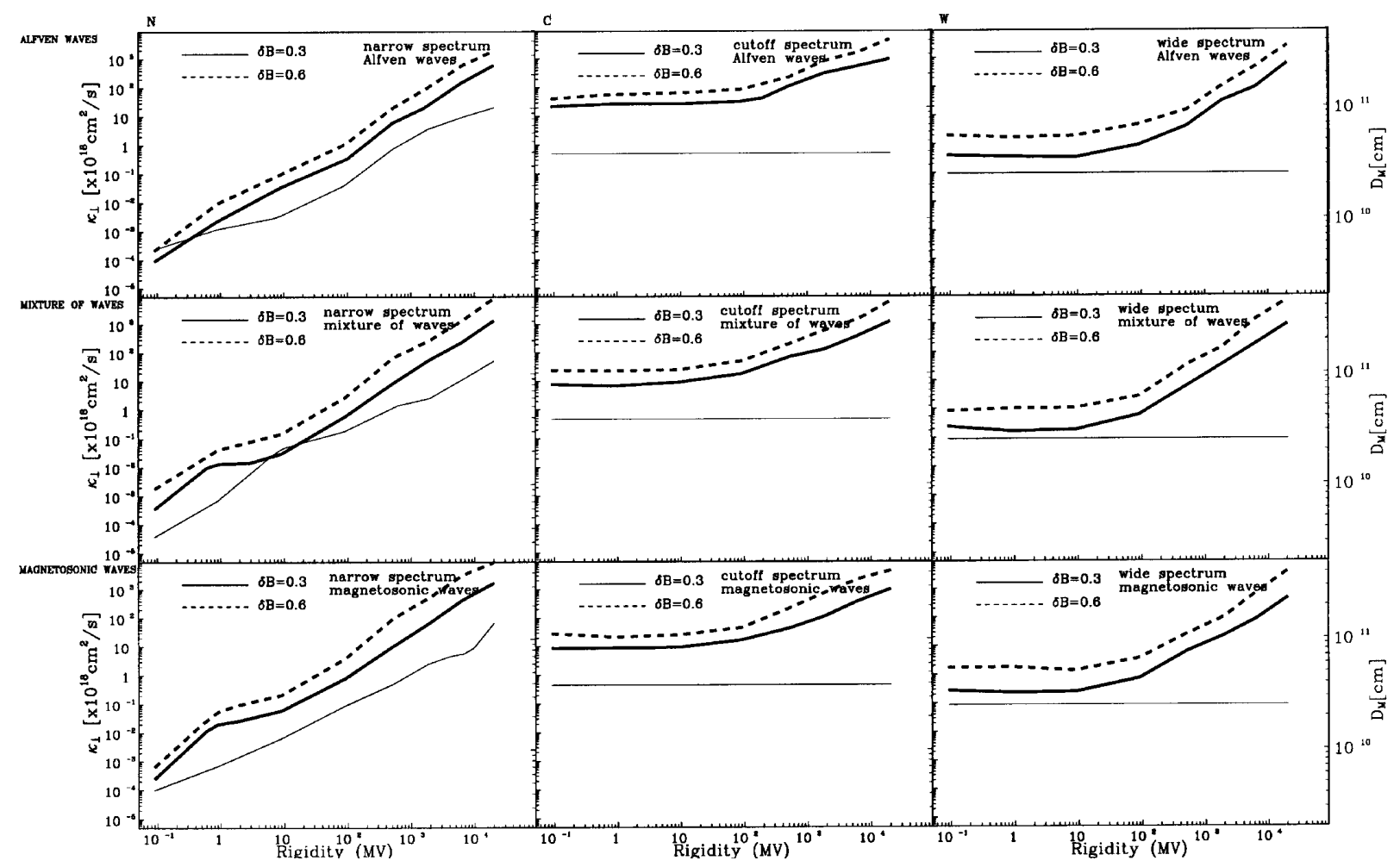

Fig. 2. The simulated values of the cross field diffusion coefficient versus particle rigidity for all considered models. From top to bottom of the picture, in the respective panels we have pure Alfvén waves, mixture of waves and pure magnetosonic waves. From left to right in the respective panels we have the $\mathrm{N}, \mathrm{C}$ and $\mathrm{W}$ spectra. The results for $\delta B=0.3$ and $\delta B=0.6$ are shown by thick solid and dashed lines, respectively. The thin solid lines indicate the diffusion coefficient of magnetic fields for models with $\delta B=0.3$. The present simulations were performed for protons.

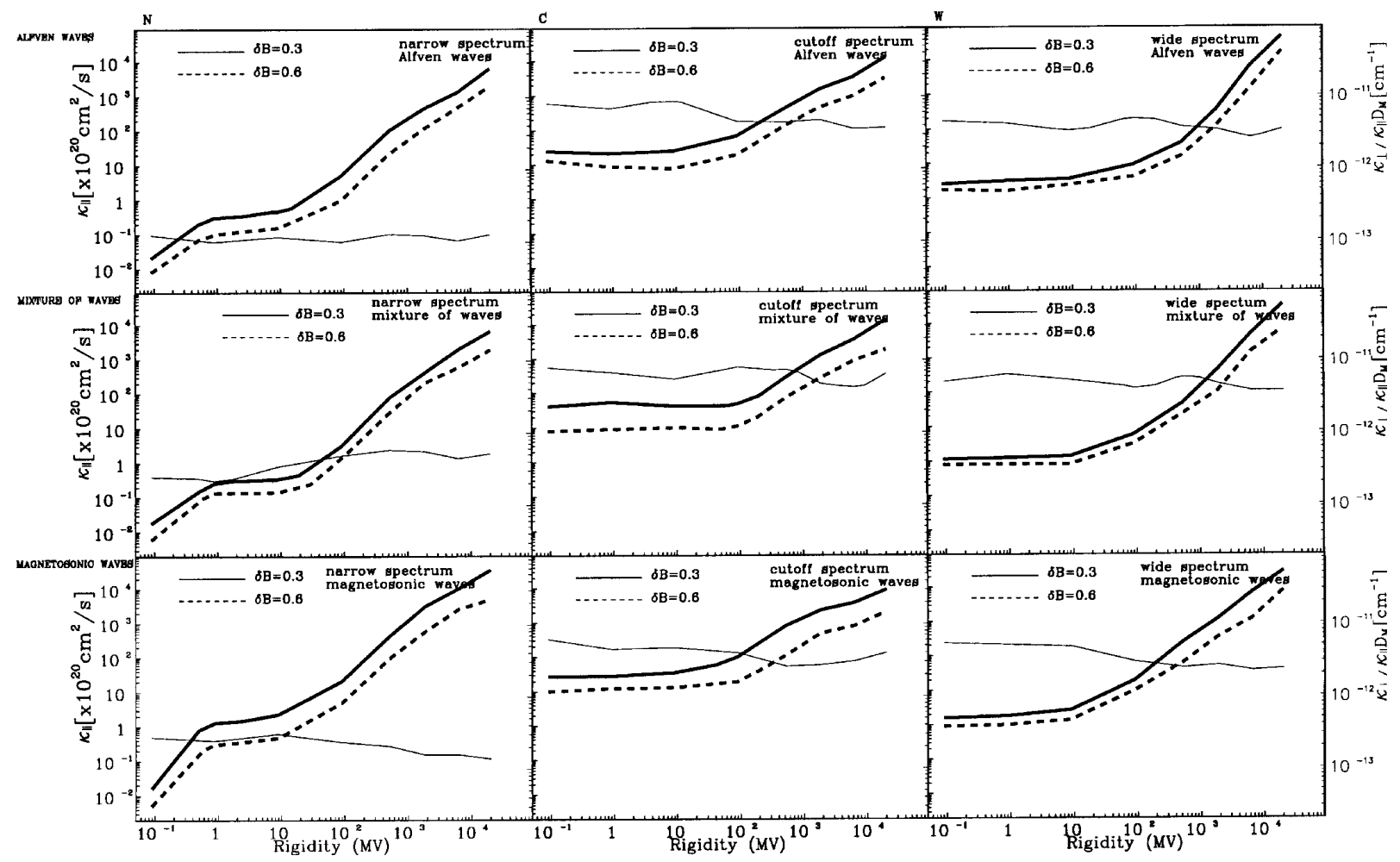

Fig. 3. The simulated values of the parallel diffusion coefficient versus particle rigidity for all considered models. From top to bottom of the picture, in the respective panels we have pure Alfvén waves, mixture of waves and pure magnetosonic waves. From left to right in the respective panels we have the $\mathrm{N}, \mathrm{C}$ and $\mathrm{W}$ spectra. The results for $\delta B=0.3$ and $\delta B=0.6$ are presented by solid and dashed lines, respectively. The thin solid lines indicate the ratio $\kappa_{\perp} / \kappa_{\|} D_{\mathrm{M}}$ for models with $\delta B=0.3$. The present simulations were performed for protons. 
interactions (more effective transport parallel to the average magnetic field), $\kappa_{\perp}$ is more than an order of magnitude larger in comparison to the models $\mathrm{N}$ and $\mathrm{W}$ and results presented by GJ99. The same situation could appear for model $\mathrm{W}$ in the low rigidity range, causing an enhancement in diffusion above predictions of the model $\mathrm{N}$ and the results presented by GJ99. It is clear that at a given value of $D_{\mathrm{M}}$ an increase in the particle parallel transport must cause a similar increase in the cross field diffusion. In the high rigidity range, transport for all the considered wave spectra models ( $\mathrm{W}, \mathrm{C}$ and $\mathrm{N}$ ) is to a similar degree controlled by resonant interactions and differences between the simulated $\kappa_{\perp}$ disappear.

We must stress that at the lowest particle velocities $\left(v \leq V_{\mathrm{A}}\right)$, where the Coulomb scattering becomes more effective, the models could be unrealistic. However, our considerations could be useful for comparison with some published results (especially for $\kappa_{\|}$) and to test models of turbulence.

\section{Final remarks}

Propagation of protons in the solar wind at a wide range of rigidities was studied for a few models of MHD waves. A choice of the turbulence model composed of Alfvén or fast magnetosonic waves relies upon the fact that these particular waves are less effectively damped in plasma with the parameter $\beta \approx 1$ and constitute a big part of the observed solar turbulence (Stix 1992; Goldstein et al. 1995). Summarizing, our simulations show that the global form of the curves of "cross field diffusion" versus rigidity is sensitive to the spectrum and amplitude of waves, but only depends to a small degree on the type of MHD waves considered. The transport across the average magnetic field could be controlled by compound diffusion to a large degree. It is shown that the field-lines random walk, together with the effective particle transport along the mean magnetic field, in the absence of resonant interactions, leads to a large enhancement of the cross field diffusion coefficient, above the results obtained by GJ99 and quasi-linear theory. It is interesting to note that the ratio $\kappa_{\perp} / \kappa_{\|} D_{\mathrm{M}} \approx$ const. is roughly independent of energy.

Acknowledgements. The work was supported by foundation of Prof. A. Krzyżanowskiego and Komitet Badań Naukowych through the grant PB 258/P03/99/17.

\section{References}

Bieber, J., Wanner, W., \& Matthaeus, W. H. 1996, JGR, 101, 2511

Duffy, P., Kirk, J. G., Gallant, Y. A., \& Dendy, R. O. 1995, A\&AS, 302, L21

Forman, M. A., Jokipii, J. R., \& Owens, A. 1974, ApJ, 192, 535

Giacalone, J., \& Jokipii, J. R. 1994, ApJ, 430, L137

Giacalone, J., \& Jokipii, J. R. 1999, ApJ, 520, 201 [三 GJ99]

Gleeson, L. 1969, Planet. Space Sci., 17, 31

Goldstein, M. L., Roberts, D. A., \& Matthaeus, W. H. 1995, ARA\&A, 33, 283

Hasselmann, K., \& Wibberenz, G. 1968, Z. Geophys., 34, 353

Jokipii, J. R. 1966, ApJ, 146, 480

Jokipii, J. R., \& Kota, J. 1999, Proc. 26th ICRC, 7, 29

Jokipii, J. R., \& Parker, E. N. 1969, ApJ, 155, 777

Kubo, R. 1957, J. Phys. Soc. Jpn., 12, 570

Michałek, G., \& Ostrowski, M. 1997, A\&AS, 326, 793

Michałek, G., \& Ostrowski, M. 1998, A\&AS, 337, 558

Michałek, G., \& Ostrowski, M. 2001, Solar Phys., in press

Karimabadi, H., \& Menyuk, C. R. 1991, JRG, 96, 9669

Ragot, B. R. 1999, ApJ, 581, 974

Schlickeiser, R. 1989, ApJ, 336, 243

Schlickeiser, R., \& Miller, J. A. 1998, ApJ, 492, 352

Stix, T. H. 1992, Waves in Plasmas (New York: AIP) 